\title{
Analysis and Discussion on the Creative Design of Architectural Visual Modeling Elements
}

\author{
Wang Dan \\ Hulunbuir university ,Hulunbuir 021008,China \\ Wang Dan(1983-),Hailar, Inner Mongolia,Lecturers,Engineer,Registered builder
}

\begin{abstract}
With the improvement of people's living standards, the socio-economic development and the advancement of urbanization, the number of construction projects has gradually increased, and people have higher and higher requirements for building quality and living environment. More and more new building materials are used in construction work, which not only enhances the quality of the building to a certain extent, but also brings a richer visual impact to the building's shape design. [1] Based on this, this article will briefly analyze the theme of "architectural visual modeling element design creativity" and analyze the application of architectural visual modeling elements in architectural engineering construction, which will bring about the role of architectural engineering construction.
\end{abstract}

\section{Introduction}

In order to ensure the beautiful appearance of the building, designers of architectural engineering need to consider various factors in the design process. Relevant research shows that the addition of architectural visual modeling elements in the construction process of the building can effectively improve the aesthetics of the building, and at the same time make the building more popular without affecting the overall quality, so as to improve the residential satisfaction of the residents. [2]

\section{Brief Discussion of Architectural Visual Modeling Elements}

\subsection{Analysis of Architectural Visual Modeling Elements}

Architectural visual modeling elements have a wide range of applications. Adding architectural visual modeling elements to the construction process, considering that the application of architectural visual modeling elements can effectively improve the aesthetics of buildings and meet people's high requirements for living environments. [3] There are many architectural visual modeling elements to choose from, among which building materials are the most important points of architectural visual modeling elements, expressing different architectural colors. According to the architectural design, choosing the appropriate building materials and architectural colors can show different visual effects, thereby continuously innovating the visual aesthetics of the building, bringing greater visual impact to the occupants, and forming different architectural visual shapes. [4] Therefore, in the process of construction project construction, in order to continuously innovate the architectural visual modeling and create richer architectural visual modeling colors, it is necessary for the architectural designer to fully consider the addition of architectural visual modeling elements during the construction design process and use them. The combination of different building materials makes the building's visual style more unique.

\subsection{Types of Architectural Visual Modeling Elements}

Concrete, brick, wood, stone, etc. are important materials for building construction, and also one of the key factors affecting the visual shape of the building. [5] With the improvement of living standards and the advancement of urbanization, people have increased the requirements for the aesthetics of buildings on the premise of ensuring living comfort, how to make the color elements of building shapes more prominent, and show the unique beauty of buildings. It has also become the primary focus of current architectural designers. Therefore, when designing a building plan, architects need to fully understand the construction situation of the construction site, select construction materials according to the construction situation, effectively integrate the construction material with the construction design drawing, and highlight the architectural style and aesthetics with different colors continuously in order to innovate the visual styling of buildings, and play the role and value of architectural design.

\subsection{Architectural Visual Modeling Element}




\section{Design Creative Role}

For architectural engineering, the creative design of architectural visual modeling elements plays an important role. It can greatly improve the quality of the project, improve the aesthetics of the building, realize the matching between the architectural engineering and the architectural design drawings, tap the unique beauty of the building. The project construction is more in line with the needs and aesthetic requirements of the development of the modern building market. The use of architectural visual modeling elements for architectural design innovation allows architectural construction to ensure both quality functions and aesthetic taste, meeting the psychological needs of the occupants. Therefore, both architects and builders should pay attention to the innovative application of architectural visual modeling elements in engineering. [6]

\section{Material Analysis of Architectural Visual Model Design}

3.1 Application of Concrete Materials in the Creative Design of Architectural Visual Modeling Elements

Concrete material is one of the main materials for the design creativity of architectural visual modeling elements. It is widely used in architectural engineering, which can make buildings have strong stability and strength, and enhance the safety of buildings. Concrete materials are mainly composed of cement, aggregate, sand, etc., and then mixed with an appropriate amount of water. Different configuration ratios will affect the performance of the final concrete. The impact of concrete on the visual shape of the building is not particularly great. Usually it needs to be matched with other architectural visual shape elements to effectively improve the aesthetics of the building, but it is the most powerful guarantee for the safe construction of the building. Nowadays, the appearance of various building materials has made the choice of architectural visual modeling elements richer. The concrete materials have also changed from the original simple selection to colored concrete, fiber concrete, glass concrete, etc., which has improved the monotonous concrete features. This can effectively improve the aesthetics of the building while ensuring the stability and strength of the building, resulting in the purpose of designing the architectural visual modeling elements. [7]

\subsection{Application of Wood in the Creative Design of Architectural Visual Modeling Elements}

Wood is also one of the important materials in the design creativity of architectural visual modeling elements. It has characteristics unmatched by other building materials, has a more natural flavor, and can bring people a sense of originality and freshness. Compared with other building construction materials, wood is lighter in texture, more malleable, and more structural. Adding wood construction to building projects can make people feel comforted. There is a kind of return to nature under the encapsulation of reinforced cement. It feels more secure and safe for residents. Especially for the construction projects with natural elements as the design point, the choice of wood for construction has unexpected effects, and it can also highlight the aesthetic beauty of its architectural visual modeling. In most ancient buildings in China, the main backbone materials were selected from wood for construction, giving the building a warmer atmosphere, making the building more affinity, and close to people's daily life. Therefore, wood is also highly selective in the creative design of architectural visual modeling elements. [8]

\subsection{Application of Bricks in the Creative Design of Architectural Visual Modeling Elements}

For reinforced concrete buildings, bricks are indispensable in the construction process. It is also one of the materials widely used in the construction process of various countries for thousands of years. The construction cost is relatively low and the inherent art is strong. Besides, it has certain classical charm, the application of bricks in large public buildings can effectively enhance the aesthetic appeal and economic attractiveness of buildings. Bricks are made of clay, cement, and other materials. The hardness is very high. In the process of making bricks, textures and different colors can be added to enrich the selectivity of the bricks so that the structural details of the building can be effectively reflected. [9]

\subsection{Application of Stone in the Design Creativity of Architectural Visual Modeling Elements}

Stone is an indispensable building material in the construction process of the past era. Even in modern times, stone is widely used in construction projects due to the positioning of the building style. Modern buildings choose stone as a building material mostly because of architectural decoration. In modern times, stone is more used as a decorative material. It has solemn, classical and other characteristics. Adding stone in the construction process can make people feel rustic and the whole building becomes solemn. Different stone bodies also have different patterns. This natural decorative line has improved the building's viewability to a certain extent, and provides many alternative materials for the creative design of architectural visual modeling elements.

\subsection{Application of Glass in the Design Creativity of Architectural Visual Modeling Elements}

With the development of social economy and technology, more and more new building materials have appeared and been applied to the design and construction of architectural engineering, which has greatly enriched the types of building materials, and the visual shape of buildings has therefore become more colorful. The glass material itself has the characteristics of transparency, smoothness, etc. The use of glass in building materials can make the entire building have a smooth and 
translucent feel, and the glass material has good light transmission, which can give a large area inside the building. The light makes the building feel transparent and bright. In the construction process of modern buildings, the use of glass materials is becoming more and more common. Most glass materials are used in the design of doors and windows, introducing a lot of light to the interior, improving the aesthetics and practicality of the building. Give occupants an impact and contribute to the design innovation of architectural visual modeling elements.

\section{Analysis of Architectural Visual Modeling Elements Design and Creative Elements}

\subsection{Application of Point Element in the Creative Design of Architectural Visual Modeling Elements}

The four elements of point, line, surface, and body are the four most basic elements in the design creativity of architectural visual modeling elements, and they are also the initial elements that make up architectural visual modeling. The composition methods are various, and different construction methods can obtain different architectural visual shapes. In architectural visual modeling element design, the point element is the least precise element. It has a certain centripetal force, but it does not have a certain shape, no clear length, width, or any area or shape. It is the smallest unit of architectural visual modeling elements. Human vision has a certain organizational ability, which can continuously simplify, abstract, and finally become a simple point. In this way, it is convenient to understand and grasp the overall pattern, and it is precisely the composition application with many small points that can form the final aesthetic pattern. During the construction process of the construction project, various small dots are moved, gathered, and combined, and different forms and patterns are given different emotions through composition. Therefore, in the creative design of architectural visual modeling elements, point elements are essential.

\subsection{Application of Line Elements in the Creative Design of Architectural Visual Modeling Elements}

If the point element is the initial element of the architectural visual modeling element design creativity, then the line element is the key element in the architectural visual modeling element design creativity. Point elements are the most simplified form of architectural visual modeling elements, while line elements are the main elements of architectural visual modeling elements. Through the combination of line elements and line elements, line elements and point elements, a certain pattern can be formed and transformed according to the ideas of architectural designers and users. Through the combination of line and other elements, the building style is highlighted. At the same time, the building is given a new form and the visual effect of the building is highlighted to achieve the creative purpose of the architectural visual modeling element design. The main combination of line elements is straight lines and curves. Curves can be infinitely changed, with certain softness, continuity, fluidity and elasticity. The curve can simply meet the design requirements of the architectural visual modeling elements, and the performance on the lines is also richer. The straight line has no stronger variability, but it has a certain sense of stability, which can make the architectural visual shape more peaceful. Therefore, the line element is also an important point in the design of architectural visual modeling elements.

\subsection{Application of Surface Elements in the Creative Design of Architectural Visual Modeling Elements}

The surface element is more prominent than the starting point element and the line element, and it can give a better sense of space to the building graphics constructed. The surface element has a wide range of applications, and it can be used not only inside the building, but also outside the building. It can make the building more in line with the public's aesthetic needs, so that the visual shape of the designed building can be both practical and aesthetic. The use of surface elements in the construction process is mainly based on the pattern content designed by the architectural designer to reconstruct the pattern. Based on the public's aesthetic needs, the pattern is reorganized to create a rich pattern content, which encourages people to see the pattern resonate, and enhance the admirability of the building and the intimacy of the building.

\subsection{Application of Body Elements in the Creative Design of Architectural Visual Modeling Elements}

Compared with the other three elements, the body element is more inclined to the collective state, breaking the two-dimensional state of the previous architectural visual modeling, and leading the architectural visual modeling from two-dimensional to three-dimensional. Common body elements include circles, cones, triangles, rectangles, squares, etc. Different body elements bring different visual impact and psychological enjoyment to the occupants. For example, the tapered body element brings a healthy and upward feeling, the round body element brings a smooth feeling, and the square and rectangular body elements bring a mature and stable feeling, which makes the architectural visual style feel richer. In the creative design of architectural visual modeling elements, builders can choose and apply body elements based on the designer's design to the aesthetics they want to express. At the same time, according to the actual situation and aesthetic needs, the body elements are changed to show the beauty of the body elements by means of simplification and expansion, so as to enhance 
the natural beauty and ecological beauty of the construction project. In this way, the building can be continuously innovated in various changes. The visual modeling element design brings a new feeling to the residents.

\section{Conclusion}

In summary, the design creativity of architectural visual modeling elements has a certain effect on the aesthetics and quality of architectural projects. Various types of new and beautiful materials have become an important part of architectural structural design. In order to effectively improve the structural strength and overall aesthetics of architectural engineering through these visual modeling elements, designers and constructors must choose the appropriate building materials according to the actual construction situation, and develop a reasonable and comprehensive architectural visual modeling scheme before construction. In this way, the buildings that are finally presented to the eyes of the public are beautiful, unique and safe.

\section{Acknowledgments}

Subject:Ministry of education industry-school cooperative education program ( 201802339022)、 Scientific research project of higher education institutions in Inner Mongolia autonomous region

(NJZY19232)

\section{References}

1. Li Zongtao. Architectural Visual Modeling Element Design Creativity [J]. Science Forum, 2018 (10): 372-372.

2. Chen Li. Creative analysis of architectural visual modeling element design [J]. Planning and Design, 2017 (08): 91-91.

3. Lv Huizi. Analysis on the creative design of architectural visual modeling elements [J]. Experience Exchange, 2019 (05): 127-128.

4. $\mathrm{Xu}$ Hailong.Analysis on the design creativity of architectural visual modeling elements [J]. China Construction Metal Structure, 2017 (06): 225-225.

5. Xiao Yulong, Liu Zhongbao. Architectural design element design creativity [J]. Science Technology and Enterprise, 2018 (02): 250-250.

6. Li Junbei.Analysis on the design creativity of architectural visual modeling elements [J]. Heilongjiang Science and Technology Information, 2018 (14): 183-183.

7. Wang Jianqi, Cao Zhong, Niu Yilin, Wu Jihai. Architectural modeling elements-the design and creativity of the framework [J]. Journal of Logistics Engineering Institute, 2015 (06): 122-123.

8. Li Xinlu, Zhao Hong, $\mathrm{Xu}$ Shiqiang. On the modeling design in architectural design [J]. Orientation to become rich through science and technology, 2019 (03): 166-167.
9. Shi Si, Xiong Qi. The application of steel materials in architectural design [J]. Forum of Science and Technology Association (second half), 2018 (01): 155-156. 